\title{
MOGE(S) nosology in low-to-middle-income countries
}

Eloisa Arbustini, Navneet Narula, G. William Dec, K. Srinath Reddy, Barry Greenberg, Sudhir Kushwaha, Thomas Marwick, Sean Pinney, Riccardo Bellazzi, Valentina Favalli, Christopher Kramer, Robert Roberts, William A. Zoghbi, Robert Bonow, Luigi Tavazzi, Valentin Fuster and Jagat Narula

In a very thoughtful News and Views article (Mayosi, B. M. MOGE(S): a standardized classification of cardiomyopathies? Nat. Rev. Cardiol. 11, 134-135 [2014]), ${ }^{1}$ Professor Mayosi described MOGE(S) as an "admirable achievement on the path towards a globally accepted nomenclature for cardiomyopathy". ${ }^{2} \mathrm{He}$ appropriately emphasized the lack of examples of aetiological notation for important cardiomyopathies from low-income and middle-income countries, such as tropical endomyocardial fibrosis (EMF; also known as tropical endomyocardial disease, constrictive endocarditis, and endocarditis parietalis fibroplastica), which is one of the most prevalent causes of restrictive cardiomyopathy. ${ }^{3}$ Mayosi suggests that an aetiological category for unexplained (or idiopathic) cardiomyopathy would improve the clinical utility of the MOGE(s) scheme. ${ }^{1}$ The authors of the scheme completely agree, and reiterate that the classification system (or nosology) is flexible, conveniently expandable, and allows easy modification of the MOGE(S) online app. ${ }^{4}$ The first update to the app has been already proposed to improve the description of arrhythmogenic right ventricular cardiomyopathy. ${ }^{5}$

EMF might provide a paradigmatic example of the flexibility of the MOGE(S) nosology and associated app. EMF is estimated to be the underlying cause of $20 \%$ of heart failure in the areas of Africa in which EMF is endemic, and is known to be the second leading cause of hospital admissions

Box 1 | MOGE(S) classifications of tropical EMF

$M_{R(E M F L V)} O_{H+\uparrow E o} G_{N} E_{\text {I-S-Schistosomiasis }} S_{C-I V}$

M Restrictive cardiomyopathy (R) associated with EMF predominantly involving the left ventricle (LV)

0 Heart involvement $(\mathrm{H})$ and $\uparrow \mathrm{Eo}$

$\mathrm{G}$ Absence of familial aggregation (N)

E Infectious aetiology (I), possibly schistosomiasis, which is suspected (S) as the cause of the EMF

S AHA stage $\mathrm{C}$ (structural heart disease with previous or current symptoms of heart failure), in NYHA functional class IV

$M_{R(E M F R V+L V)} O_{H+L i+\uparrow E O} G_{N} E_{1-U} S_{C-I I I}$

$M$ Restrictive cardiomyopathy $(R)$ associated with EMF involving both the right ventricle (RV) and the left ventricle (LV)

0 Heart $(\mathrm{H})$ and liver $(\mathrm{Li})$ involvement and $\uparrow$ Eo

$\mathrm{G}$ Absence of familial aggregation (N)

E Possible infectious aetiology (I), which is actually unknown (U)

$S$ AHA stage $\mathrm{C}$, in NYHA functional class III

$M_{R(E M F R V)} O_{H+L i+\uparrow E O} G_{N} E_{\text {I-S-Helminthiasis }} S_{C-I V}$

$M$ Restrictive cardiomyopathy $(\mathrm{R})$ associated with EMF predominantly involving the right ventricle (RV)

0 Heart $(\mathrm{H})$ and liver (Li) involvement and $\uparrow$ Eo

$\mathrm{G}$ Absence of familial aggregation (N)

E Infectious aetiology (I), possibly helminthiasis, which is suspected (S) as the cause of the EMF

S AHA stage $\mathrm{C}$, in NYHA functional class IV

$M_{R \text { (EMF LV) }} \mathrm{O}_{\mathrm{H}+\uparrow \mathrm{AO}_{0}} \mathrm{G}_{\mathrm{N}} \mathrm{E}_{\text {EO-HES-PDGFRA/PDGFRB }} \mathrm{S}_{\mathrm{C}-\| I}$ *

$\mathrm{M}$ Restrictive cardiomyopathy (R) associated with EMF predominantly involving the left ventricle (LV)

0 Heart $(\mathrm{H})$ involvement and $\uparrow$ Eo

$\mathrm{G}$ Absence of familial aggregation (N)

E Eosinophilic heart disease (Eo) and hypereosinophilic syndrome (HES), in which a PDGFRA/ PDGFRB fusion gene has been identified

S AHA stage $C$, in NYHA functional class II

*This example is provided to describe the EMF variety associated with hypereosinophilic syndrome and mutations in oncogenic tyrosine kinase receptors. Abbreviations: EMF, endomyocardial fibrosis; $\uparrow$ Eo, hypereosinophilia.

for acquired cardiovascular disease in children and young adults, after rheumatic heart disease. ${ }^{6}$ However, the aetiology of EMF remains elusive. Several hypotheses have been proposed, such as cardiotoxicity of eosinophils and hypereosinophilia ( $\uparrow \mathrm{Eo}$ ), often associated with parasitic infectious aetiology (schistosomiasis, Loa loa microfilaria, filariasis, or malaria), autoimmunity (high titres of malarial antibodies, hyperimmune malarial splenomegaly, raised antistreptolysin titres in patients with EMF), genetic or ethnic predisposition (high incidence among particular ethnic groups in some countries), diet (tuber cassava, tapioca) in association with extreme deprivation of proteins (tryptophan deficiency), and geochemical and climate characteristics (caesium derived from monazite soils). ${ }^{7-14}$ Although none of these factors has been proven to cause EMF, the MOGE(S) nosology can be invoked as follows.

The condition can manifest as isolated or dominant left ventricular (LV) EMF, isolated or dominant right ventricular (RV) EMF, or biventricular ( RV + LV) EMF, and can be stated as such in the MOGE(S) description. MOGE(S) can also describe the organ involvement in addition to the heart $(\mathrm{H})$, such as liver involvement $(\mathrm{H}+\mathrm{L})$, or $\uparrow$ Eo. Aetiology can be listed as being known (K), suspected (S), or unknown (U). If known or suspected, the aetiology can be coupled with description of the injurious agent. Although isolated EMF is easily described by MOGE(S), the presence of $\uparrow$ Eo goes beyond the cardiac evaluation and requires haematological and immunological investigation and classification. Various classifications of $\uparrow$ Eo disorders and associated syndromes have been formulated in the past, and the Year 2011 Working Conference on Eosinophil Disorders and Syndromes refined the criteria and definitions. ${ }^{15}$ The description of EMF with $\uparrow$ Eo, with or without involvement of organs other than the heart (such as lungs, skin, liver, gastrointestinal tract, pancreas, or kidneys) 
requires a multidisciplinary diagnostic work-up, starting with elucidation of the cause of $\uparrow$ Eo. The important information for treatment is the identification of fusion genes involving PDGFRA, PDGFRB, and FGFR1. $\operatorname{MOGE}(S)$ can describe the mutations in oncogenic tyrosine kinase receptors in the aetiology section, or their absence (negative), or the lack of information (not tested).

The examples in Box 1 were generated using the MOGE(S) app. ${ }^{4}$ Therefore, MOGE(S) and its app can readily provide a description for any type of cardiomyopathy, with the advantage of integrating important information that can constitute the basis for a novel, worldwide epidemiology to describe diseases, not only on the basis of their clinical phenotypes, but also according to their aetiology.

Center for Inherited Cardiovascular Diseases, Pavia, Italy (E.A., V. Favalli). Department of Pathology, New York Presbyterian Hospital-Weill Cornell Medical College, New York, NY, USA (N.N.). Cardiology Division, Massachusetts General Hospital, Boston, MA, USA (G.W.D.). Public Health Foundation of India, New Delhi, India (K.S.R.). University of California at San Diego, San Diego, CA, USA (B.G.). Mayo Clinic College of Medicine, Rochester, MN, USA (S.K.). University of Queensland, Princess Alexandra Hospital, Queensland, Australia (T.M.). Mount Sinai Medical Center, One Gustave L. Levy Place, New York, NY 10029, USA (S.P., V. Fuster, J.N.). Department of Industrial and
Information Engineering, University of Pavia, Pavia, Italy (R. Bellazzi). University of Virginia, Charlottesville, VA, USA (C.K.). John \& Jennifer Ruddy Canadian Cardiovascular Research Center, University of Ottawa Heart Institute, Ottawa, ON, Canada (R.R.). Methodist DeBakey Heart \& Vascular Center, The Methodist Hospital, Houston, TX, USA (W.A.Z.). Center for Cardiovascular Innovation, Chicago, IL, USA (R. Bonow). Maria Cecilia Hospital, GVM Care \& Research, E.S., Health Science Foundation, Cotignola, Italy (L.T.).

Correspondence to: J.N.

narula@mountsinai.org

\section{Competing interests}

B.G. is a consultant for Celladon, Mesoblast, Novartis, Teva Pharmaceutical, and Zensun; and is on the speakers' bureau of Boehringer Ingelheim and Otsuka. C.K. has received research support from Novartis (research funding) and Siemens Healthcare (equipment); and is a consultant for St. Jude Medical and Synarc. J.N. has received research support from GE Healthcare and Phillips (equipment to the institution from both). The other authors declare no competing interests.

1. Mayosi, B. M. Cardiomyopathies: MOGE(S): a standardized classification of cardiomyopathies? Nat. Rev. Cardiol. 11 134-135 (2014).

2. Arbustini, E. et al. The MOGE(S) classification for a phenotype-genotype nomenclature of cardiomyopathy: endorsed by the World Heart Federation. J. Am. Coll. Cardiol. 62, 2046-2072 (2013).

3. Sliwa, K. \& Mocumbi, A. O. Forgotten cardiovascular diseases in Africa. Clin. Res. Cardiol. 99, 65-74 (2010).

4. Biomeris. MOGES [online], http:// moges.biomeris.com/moges.html (2014).
5. Arbustini, E. et al. Reply to Letter to the Editor: the MOGE(S) classification for a phenotypegenotype nomenclature of cardiomyopathy: more questions than answers? J. Am. Coll. Cardiol. (in press).

6. Freers, J., Mayanja-Kizza, H., Ziegler, J. L. \& Rutakingirwa, M. Echocardiographic diagnosis of heart disease in Uganda. Trop. Doct. 26, 125-128 (1996).

7. Carneiro Rde, C. et al. Endomyocardial fibrosis associated with mansoni schistosomiasis. Rev. Soc. Bras. Med. Trop. 44, 644-645 (2011).

8. Berenguer, A., Plancha, E. \& Muñoz Gil, J. Right ventricular endomyocardial fibrosis and microfilarial infection. Int. J. Cardiol. 87, 287-289 (2003).

9. Andy, J. J. Aetiology of endomyocardial fibrosis (EMF). West Afr. J. Med. 20, 199-207 (2001).

10. Wayengera, M. Searching for new clues about the molecular cause of endomyocardial fibrosis by way of in silico proteomics and analytical chemistry. PLOS ONE 4, e7420 (2009).

11. Mayosi, B. M. \& Somers, K. Cardiomyopathy in Africa: heredity versus environment. Cardiovasc. J. Afr. 18, 175-179 (2007).

12. Sezi, C. L. Effect of protein deficient cassava diet on Cercopithecus aethiops hearts and its possible role in the aetiology and pathogenesis of endomyocardial fibrosis in man. East Afr. Med. J. 73 (Suppl.), S11-S16 (1996).

13. McKinney, B. Studies on the experimental production of endomyocardial fibrosis and cardiomegaly of unknown origin by dietary means. Am. Heart J. 90, 206-214 (1975).

14. Kuruvilla, L. \& Kartha, C. C. Cerium depresses endocardial endothelial cellmediated proliferation of cardiac fibroblasts. Biol. Trace Elem. Res. 114, 85-92 (2006).

15. Valent, P. et al. Contemporary consensus proposal on criteria and classification of eosinophilic disorders and related syndromes. J. Allergy Clin. Immunol. 130, 607-612 (2012). 